\title{
Estimativa da produção de uma lavoura de agricultura familiar através de imagens digitais capturadas por veículo aéreo não tripulado (VANT)
}

\author{
Gerson L. Serejo ${ }^{1}$, Viviane A. Santos ${ }^{1}$, Ana C. S. Gomes ${ }^{2}$, Alexandre F. B. Silva ${ }^{1}$ \\ ${ }^{1}$ Universidade Federal do Pará, Campus Tucuruí, Rodovia BR 422, Km 13, Canteiro de \\ Obras - UHE, Tucuruí, Pará, Brazil. \\ ${ }^{2}$ Instituto Senai de Inovação - ISI, Av. Brás de Aguiar, nº 548, Belém, Pará, Brazil. \\ gerson.serejo@tucurui.ufpa.br, vsantos@ufpa.br, \\ claudia.isi@senaipa.org.br, alexandre.fb.silva@gmail.com
}

\begin{abstract}
Family farming has become an important alternative means of ensuring the sustainability of food production systems in Brazil. At Tucurui/PA there are a lot of farms of this type but they lack information to assist them in decision making. The objective of this work is to estimate the harvesting of cassava by a small producer in the region, by employing digital image processing (DIP) and computer vision (CV) techniques.
\end{abstract}

Resumo. A agricultura familiar tem se tornado uma alternativa importante para garantir a sustentabilidade dos sistemas de produção de alimentos no Brasil. Em Tucurui/PA, existem muitas fazendas deste tipo, mas carecem de informações que auxiliem na tomada de decisões. O objetivo deste trabalho é estimar a colheita de mandioca de um pequeno produtor da região, através do emprego de técnicas de processamento digital de imagens (PDI) e visão computacional (VC).

\section{Introdução}

A agricultura familiar consiste no cultivo de terras em pequenas propriedades por membros da família. Sua importância para o sistema global de produção de alimentos é enorme. No Brasil, cerca de $70 \%$ da produção de alimentos vem da agricultura familiar e não da agricultura em grande escala [SEAD 2017]. Este sistema tem um efeito positivo na produção agrícola, pois beneficia o solo e reduz o desperdício de recursos naturais. Também tem implicações sociais porque leva a um maior crescimento de empregos e rendas do que em grandes empresas rurais modernizadas [CONTAG 2017].

O município de Tucuruí, no estado do Pará, faz parte de uma região de agricultura familiar. Essa região se destaca pelo plantio de mandioca na época das chuvas [FAPESPA 2015]. Segundo a Empresa Brasileira de Pesquisa Agropecuária EMBRAPA (2018), a Região Norte do Brasil é a maior produtora dessa cultura com $36,1 \%$ da produção nacional. No Pará, $60,66 \%$ das terras são cultivadas e isso responde por $56,96 \%$ da produção da região. Porém, mesmo ocupando grandes áreas plantadas e tendo atualmente a maior produção de mandioca do País, ocupa apenas a $4^{\mathrm{a}}$ posição em produtividade, com uma produção de $1,5 \mathrm{~kg} / \mathrm{m}^{2}$, contra $2,2 \mathrm{~kg} / \mathrm{m}^{2}$ alcançada pelo primeiro lugar. Vários fatores são responsáveis pela baixa produtividade agrícola do Pará, mas a principal causa é a forma de produção artesanal predominante. 
Este estudo exploratório, realizado em Tucuruí, buscou estimar a produtividade de uma safra de mandioca. Há uma escassez de estudos de PDI e VC em lavouras de mandioca, principalmente quando se trata de estudos envolvendo imagens adquiridas por VANT. A pesquisa fornece detalhes de como os resultados foram obtidos por meio de índices de vegetação, como: Excess Green (ExG) [Woebbecke et al. 1995], Excess Green Minus Excess Red (ExGR) [Meyer et al. 2004] e o Maximum Green (MaxG) [Moorthy et al. 2015], aplicados às imagens. Como contribuição científica, este estudo provê um processo para a obtenção da produtividade agrícola que considera: procedimentos de pré-processamento de imagens, seleção de índices de vegetação, segmentação de imagens, avaliação da acurácia dos índices, reconhecimento e contagem de mudas. $\mathrm{O}$ algoritmo utilizado na contagem automática está disponível nos códigosfonte do projeto ${ }^{1}$ para consulta e pesquisas futuras.

Este artigo está organizado como segue: na Seção 2 são apresentados os trabalhos relacionados; na Seção 3, a metodologia aplicada no estudo é descrita; na Seção 4 são apresentados os resultados e as discussões; e na Seção 5, as conclusões.

\section{Revisão da Literatura}

O processo de aquisição de imagens digitais de VANT para tomada de decisão está se tornando mais acessível e fornecendo resolução de melhor qualidade do que imagens geradas por satélite [Anguiano e Morales et al. 2018]. VANTs também fornecem uma alternativa para as plataformas de detecção de solo e resolução espacial permitindo a extração de informações em escala [Varela et al. 2018]. Com o auxílio de imagens aéreas digitais, é possível aplicar as técnicas de PDI e VC para separar o verde das imagens de forma mais clara e, assim, permitir a contagem das mudas, de forma que uma estimativa de sua produção pode ser feita posteriormente. Uma das principais técnicas baseadas em imagens empregadas na agricultura é conhecida como segmentação, que envolve a aplicação de índices de vegetação para separar plantas (colheitas e ervas daninhas) de seu fundo (solo e resíduos).

Hamuda et al. (2016) fizeram uma revisão dos principais índices verdes considerados adequados para segmentação de plantas, que são: Normalized Difference Index (NDI) [Woebbecke et al. 1993], Excess Green Index (ExG) [Woebbecke et al. 1995], Excess Red Index (ExR) [Meyer et al., 1998] [Meyer et al. 1999], The Color Index of Vegetative Extraction (CIVE) [Kataoka et al. 2003], Excess Green Minus Excess Red (ExGR) [Meyer et al. 2004], Normalized Green Red Difference Index (NGRDI) [Hunt et al. 2005], Vegetative Index (VEG) [Hague et al. 2006]. Eles conduziram uma avaliação detalhada discussão sobre pré-processamento e segmentação, e destacou os desafios de envolver métodos de limiar para as condições complexas que podem ser encontradas em campos agrícolas.

Gnädinger e Schmidhalter (2017) usaram técnicas PDI para imagens capturadas a $50 \mathrm{~m}$ de altura com um octocóptero equipado com câmera de $10 \mathrm{MP}$ capturando imagens a cada 5 segundos. Foi utilizado um procedimento de realce de contraste, o que melhorou a diferenciação das cores nas imagens. Em média, o erro entre as plantas contadas visualmente e digitalmente foi $\leq 5 \%$. A cobertura do solo, conforme determinado pela análise de pixel verde, variou de $76 \%$ a $83 \%$ nessas fases. No entanto,

\footnotetext{
${ }^{1}$ https:/github.com/gerson-serejo/matlab
} 
a correlação entre a cobertura do solo e as plantas contadas digitalmente foi muito baixa. A presença de ervas daninhas e o efeito borrado nas imagens representaram possíveis erros na contagem de plantas.

Netto et al. (2018) também avaliaram os índices de vegetação mencionados acima (ExG, ExGR e NDI) para o desempenho de três métodos de limiarização automatizados Otsu, Riddler [Riddler 1978] e Triangle [Zack et al. 1977] em termos de precisão para uma cultura de milho. Os resultados mostraram que o método Triangle teve um desempenho superior quando precedido pelos índices NDI (90,70\%) e ExGR $(90,23 \%)$ e os métodos Otsu e Riddler quando precedido pelo NDI com taxas de acurácia de $89,06 \%$ e $89,03 \%$, respectivamente.

Varela et al. (2018) desenvolveu um método para contar plantas de milho usando o índice de vegetação ExG. Eles aplicaram aprendizado de máquina ao método usando um procedimento de treinamento escalonável com dois modos de treinamento. O método de precisão aplicado atingiu $96 \%$, quando a resolução da imagem atingiu altitudes de $2,4 \mathrm{~m}$ e $10 \mathrm{~m}$.

Ghosal et al. (2019) usaram uma rede neural convolucional (CNNConvolutional Neural Network) para detectar e contar plantas com cabeça de sorgo. Eles demonstraram que é possível reduzir o trabalho necessário para a rotulagem humana sem prejudicar o desempenho do modelo final usando um modelo semitreinado da CNN. Ao adotar sua abordagem, os usuários podem ter uma melhor compreensão do aprendizado profundo treinado e ser capazes de confiar em suas decisões. Outra técnica também foi utilizada para esse fim, que foi a representação gráfica em 3D, aplicada por Feng et al. (2019); este processou imagens ortomosaicas e um modelo digital de elevação para medir a altura da planta. Eles também conduziram uma análise de correlação para estimar os rendimentos com erros aceitáveis.

Além disso, algoritmos específicos foram criados para estimar com precisão a densidade da cultura junto as ervas daninhas. McCraine et al. (2019) usou a abordagem baseada na transformada de Hough. Os resultados mostraram que seu grau de acurácia foi de $82,3 \%$, o que reforça a necessidade de aprimorá-lo.

Os trabalhos acima mencionados confirmam que existem soluções neste domínio, embora seja necessário ultrapassar o problema dos erros de contagem e garantir a precisão. Meyer, Camargo e Neto (2008) explicaram que existem dois problemas não resolvidos em relação à pesquisa do índice de vegetação, que são a falta de uma definição clara do tipo de limiar (manual ou automático) que deve ser aplicado durante a fase de binarização da imagem, e a falta de evidências sobre a precisão do índice de vegetação. A maioria das pesquisas examinadas nesta seção é baseada na seleção de limiar, seja manualmente, selecionando um tom de verde de intensidade variável (a análise de histograma), ou automaticamente usando métodos de limiar. Outra preocupação é como validar as soluções atuais envolvendo diversos fatores, como as condições de campos agrícolas reais, altura da imagem, intensidade da cor, etc.

\section{Metodologia}

Nesta seção são apresentadas a área do estudo, os materiais utilizados e os procedimentos metodológicos realizados. 


\section{1 Área de estudo}

A Figura 1 (a) mostra a localização da Fazenda Bela Vista, que possui área total de $23.000 \mathrm{~m}^{2}$ e suas características de relevo são de morro. As coordenadas da fazenda são $3^{\circ} 39^{\prime} 17,0$ "S $49^{\circ} 46^{\prime} 32,7^{\prime \prime} \mathrm{W}$.

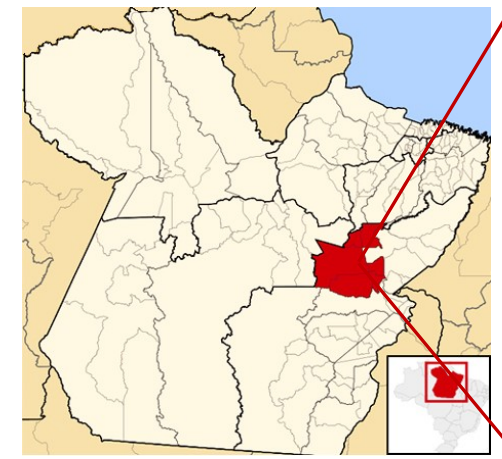

(a)

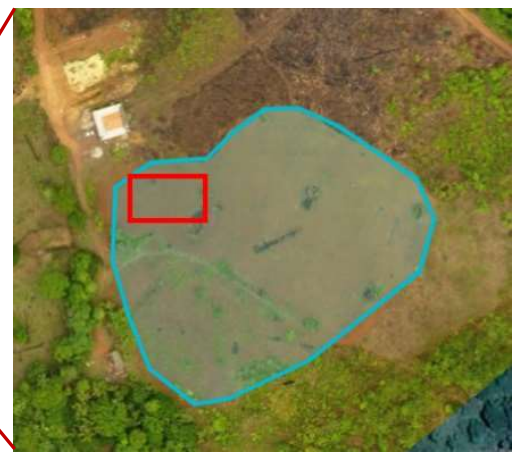

(b)

Figura 1 - (a) Localização da Fazenda Bela Vista. (b) Área total (azul) e área selecionada (vermelho) da plantação.

A Figura 1 (b) exibe a área da plantação em azul com dimensão de $12.234 \mathrm{~m}^{2}$. Já a área em vermelho possui $327 \mathrm{~m}^{2}$ e foi escolhida para a aplicação dos índices de vegetação neste estudo, pois possui o melhor preparo do solo, de acordo com $\mathrm{o}$ agricultor, e uma área mais plana, com menos desníveis possíveis e linhas de plantio mais definidas.

\subsection{Aquisição de imagens}

As imagens foram capturadas por um $\mathrm{VANT}^{2}$ em visitas programadas à fazenda. Todos os voos foram realizados de forma automática.

\section{3. Índices de vegetação escolhidos}

Para identificar as mudas de mandioca na imagem, foram aplicados três índices de vegetação: ExG que é um dos mais usados na literatura para destacar o verde das imagens; ExGR que tem se tornado um índice bastante eficiente para plantações; e MaxG que consiste na separação dos pixels da cor verde.

\subsection{Segmentação}

Esta técnica é usada para separar partes de interesse de uma imagem [Schalkoff 1989], como a separação da vegetação do solo. O método mais utilizado na agricultura é a limiarização, que consiste em segmentar a imagem, escolhendo um ponto de corte.

No estudo, a limiarização para cada índice de vegetação foi aplicada por meio de seleção automática e manual (Figura 2). A seleção automática baseou-se no Método Otsu [Otsu 1979], enquanto a seleção manual baseou-se em uma escolha empírica, que foi feita após alguns testes visuais, onde o limiar estava mais próximo do tom da cor da plantação de mandioca. A nomenclatura usada para a seleção manual de limiar (SML) foi: ExG + SML, ExGR + SML e MaxG + SML. Já os índices que utilizaram o

\footnotetext{
${ }^{2}$ DJI Phantom 4 com peso de $1.388 \mathrm{~g}$, autonomia de cerca de 25 minutos por bateria, velocidade máxima
} de $72 \mathrm{~km} / \mathrm{h}$, câmera RGB de resolução de $12 \mathrm{MP}$ estabilizada por um GIMBAL tri-axial. 
método Otsu foram designados como ExG + Otsu, ExGR + Otsu e MaxG + Otsu. Após a limiarização foi realizada a binarização da imagem.

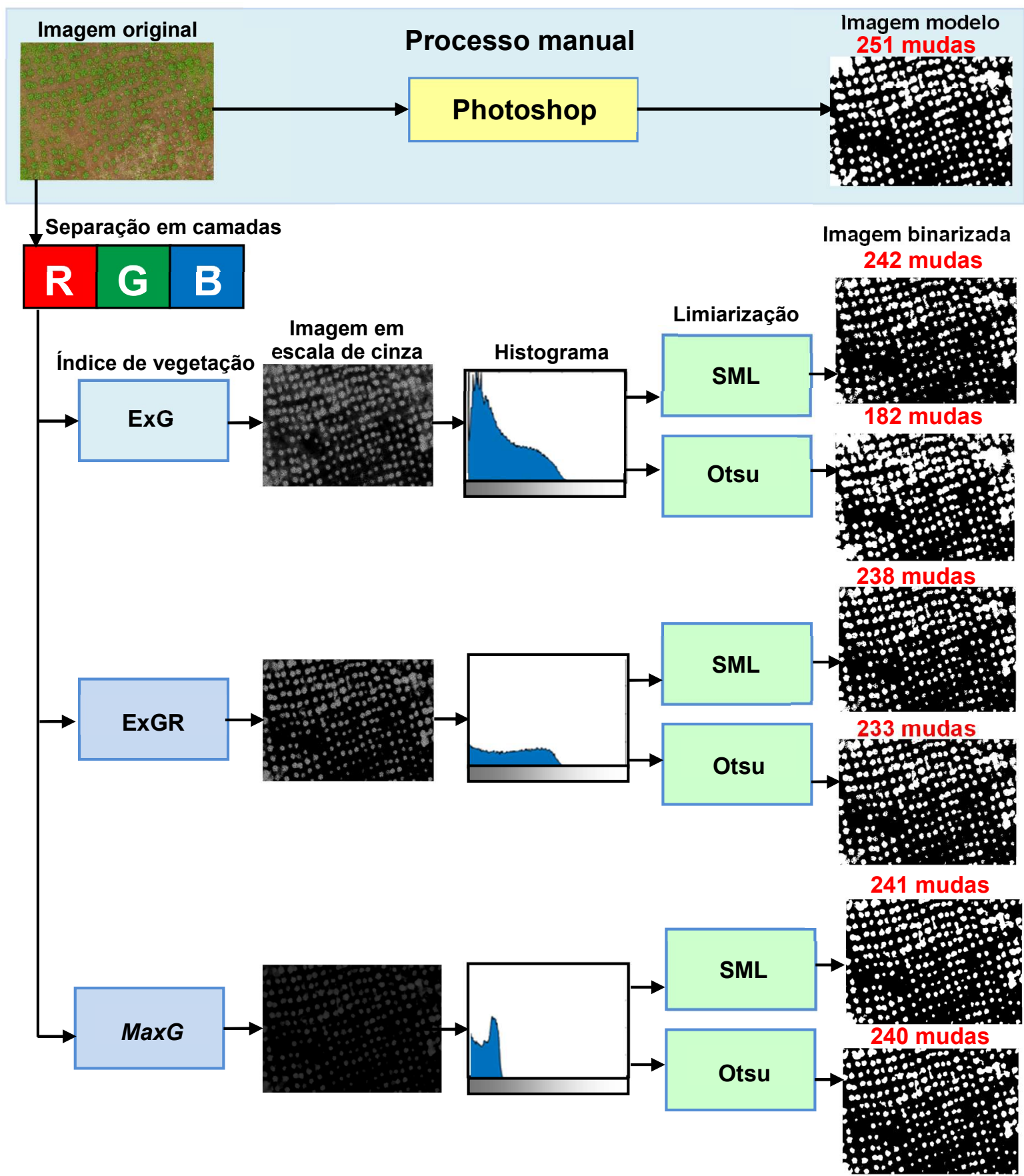

Figura 2 - Fluxograma das etapas do processo de contagem automática em comparação com o processo manual.

\subsection{Avaliação da acurácia dos índices}

Avaliou-se a acurácia dos índices de vegetação através do fator de qualidade definido pelo ATRWG e utilizado por Meyer e Camargo - Neto (2008). Na avaliação deste fator de qualidade, quanto mais próximo de 1,0 melhor é a separação de todos os pixels de classe selecionados. Próximo de zero representa uma pior separação dos pixels em 
relação a imagem B. O fator de qualidade foi aplicado a cada planta extraída da binarização automática e manual.

A Figura 2 apresenta de maneira esquemática as etapas do pré-processamento, aplicação dos índices de vegetação, histogramas, limiarização, binarização e contagem das mudas nas imagens. A imagem gerada pelo processo manual ${ }^{3}$ foi utilizada na comparação dos resultados. O número total de mudas para o processo manual (251 mudas) conforme a Figura 2 serve como comparação para o processo de contagem automática.

\subsection{Estimativas}

Para a contagem das mudas de mandioca foi feita a estimativa de mudas para a área segmentada (Figura 2) e depois para a área total, através de uma regra de três simples. De acordo com a EMBRAPA (2018), cada muda de mandioca pode gerar em média até $1,5 \mathrm{~kg}$ de raiz. Portanto, com a contagem total de mudas da plantação, multiplicou-se pelo peso médio em $\mathrm{kg}$ da raiz produzida por cada muda para se estimar a produção.

\section{Resultados e Discussão}

Os valores para os índices de vegetação foram calculados a partir dos histogramas apresentados na Tabela 1. Nos histogramas percebeu-se que a alta incidência de curvas acentuadas causa maior interferência na luminosidade e, assim, leva a uma maior gama de cores no espectro. Isso significa que existe a necessidade de uma seleção de limiares para melhorar a quantificação da cultura, conforme apontado por Soares (2017).

O histograma do ExG tem uma curva acentuada, o que significa que este índice reconheceu mais tons de verde do que ExGR e MaxG. Na Tabela 1, ExG+SML e ExG+Otsu alcançaram valores superiores de 0,29 e 0,25 respectivamente, para os tons verdes detectados. Esse resultado está de acordo com o encontrado na literatura [Hamuda et al. 2016; Moorthy et al. 2015; Meyer e Camargo-Neto 2008], uma vez que comparações envolvendo ExG para separação de plantas e não-plantas apresentaram resultado satisfatório.

A taxa de acurácia foi de aproximadamente $83 \%$ para ExG+SML, o que é satisfatório para separação de vegetação. O ExGR+Otsu também demonstrou um bom índice de qualidade de segmentação de $78 \%$, próximo ao encontrado na literatura [Hamuda et al. L2016; Meyer e Camargo-Neto 2008]. MaxG+Otsu apresentou a menor acurácia de $69 \%$. No entanto, MaxG+SML ultrapassou a qualidade de segmentação do ExGR. A razão de acurácia obtida neste estudo é comparável a de outros estudos que adotam métodos semelhantes. Por exemplo, Varela (2018) obteve 96\% para ExG na imagem original e este diminuiu para $89 \%, 85 \%$ e $68 \%$, respectivamente, após a redução da resolução da imagem.

$\mathrm{Na}$ Figura 2, pode-se observar que a imagem binarizada do ExG+SML, proporcionou um delineamento mais claro do formato das folhas da mandioca, o que difere das imagens binarizadas do ExGR e MaxG. Assim, nesta condição, o ExG estaria mais sujeito a auxiliar na detecção do formato das folhas da mandioca.

\footnotetext{
${ }^{3}$ Este processo foi feito no Adobe Photoshop C S6 (13.0) aplicando-se o filtro de ajuste de contraste $($ valor $=35)$ e brilho $($ valor $=0)$.
} 
Devido a limiarização do ExGR e MaxG não ter se mostrado significativo para a interpretação das respectivas imagens resultantes, a contagem das mudas para a análise empírica e automática do histograma para estes índices foram próximas, o que sugere a possibilidade de ser desnecessário o uso de limiarização nestes tipos de índice, pois a acurácia pode independer da seleção do limiar. Meyer e Camargo - Neto (2008) denominam este tipo de situação de limiar zero incorporado, ou seja, não precisa de definição de limiar para a interpretação da imagem. Isto permite que um sistema de VC seja não-supervisionado na definição do limiar.

Com relação aos índices aplicados, o ExG obteve o melhor desempenho para a quantificação das mudas ao obter uma taxa de contagem de $96,4 \%$, seguido do MaxG que contabilizou $96 \%$ das mudas de mandioca. Como as plantas estavam em estágio de germinação no momento da coleta das imagens, ExG foi provavelmente o melhor porque ainda havia muito solo na imagem. Como os autores do índice ExG e outros estudos recentes afirmam [Guerrero 2012; Hamuda 2016], ExG é a melhor escolha para separar plantas do solo nu em condições de campo.

Tabela 1 - Valores dos índices aplicados

\begin{tabular}{|c|c|c|c|c|c|c|c|}
\hline Índice & Histograma & Método & $\begin{array}{c}\text { Valor } \\
\text { do } \\
\text { Índice }\end{array}$ & $Q_{\text {seg }}$ & $\begin{array}{c}\% \\
\text { Acurácia }\end{array}$ & $\begin{array}{l}\text { Contagem } \\
\text { de Mudas }\end{array}$ & $\begin{array}{c}\text { Taxa de } \\
\text { contagem }\end{array}$ \\
\hline \multirow[t]{2}{*}{ ExG } & & SML & 0,29 & 0,8280 & $83 \%$ & 242 & $96,4 \%$ \\
\hline & & Otsu & 0,25 & 0,8067 & $81 \%$ & 182 & $72,5 \%$ \\
\hline \multirow[t]{2}{*}{ ExGR } & 4000 r & SML & 0,24 & 0,7250 & $73 \%$ & 238 & $94,8 \%$ \\
\hline & & Otsu & 0,21 & 0,7770 & $78 \%$ & 233 & $92,8 \%$ \\
\hline \multirow[t]{2}{*}{ MaxG } & & SML & 0,05 & 0,7529 & $75 \%$ & 241 & $96 \%$ \\
\hline & & Otsu & 0,07 & 0,6882 & $69 \%$ & 240 & $95,6 \%$ \\
\hline & o & & & & & & \\
\hline
\end{tabular}

Após a quantificação das mudas, foi feita uma estimativa da produção (Tabela 2) da colheita em toneladas. Ao final da safra, a produção reportada pelo agricultor foi de 14 toneladas, valor próximo ao estimado para o processo manual. Assim, o índice ExG obteve 13,5 toneladas e obteve o melhor resultado para a fixação da imagem em estudo, ao contar 96,4\% das mudas com índice de acurácia de 83\%. O MaxG também obteve um resultado promissor com uma taxa de contagem de $96 \%$ das mudas, mas a taxa de acurácia foi menor, o que significa que é necessário melhorar esta taxa. As taxas de contagem poderiam ser aprimoradas com a aplicação de métodos de processamento de imagem morfológica para desconectar plantas acopladas, conforme percebido nas imagens binarizadas da Figura 2. 
Tabela 2 - Estimativas totais de mudas e da produção

\begin{tabular}{l|c|c}
\hline Índice & $\begin{array}{c}\text { Número total de mudas } \\
\text { (Na área total } \mathbf{1 2 . 2 3 4} \mathbf{~ m}^{\mathbf{2}} \text { ) }\end{array}$ & $\begin{array}{c}\text { Estimativa da produção } \\
\text { em toneladas }\end{array}$ \\
\hline Contagem manual & 9287 & 14.0 \\
\hline ExG+SML & 8954 & 13.5 \\
\hline ExG+Otsu & 6734 & 10.0 \\
\hline ExGR+SML & 8806 & 13.2 \\
\hline ExGR+Otsu & 8621 & 13.0 \\
\hline MaxG +SML & 8917 & 13.4 \\
\hline MaxG +OTSU & 8880 & 13.3 \\
\hline
\end{tabular}

Os resultados sugerem que as imagens baseadas em VANT têm potencial para quantificar plantas e estimar a produção agrícola com uma taxa de erro satisfatória de $17 \%$ para ExG, 25\% para MaxG e $27 \%$ para ExGR, o que é aceitável, conforme mencionado por Guijarro (2011).

\section{Conclusões}

Esta pesquisa adotou técnicas de pré-processamento, aplicação de índices de vegetação, segmentação, extração e interpretação de imagens capturadas por um VANT. As imagens foram utilizadas para quantificar mudas de mandioca e estimar sua produtividade em uma fazenda de pequeno porte em Tucuruí/PA, localizada na região amazônica que se caracteriza por este tipo de atividade fundiária. Os procedimentos adotados proporcionaram resultados satisfatórios, quando comparados com a quantificação final da safra e estimativa de produtividade do próprio agricultor.

$\mathrm{Na}$ etapa de aquisição da imagem, notamos a importância da seleção de uma área representativa que servirá de linha de base para a estimativa final. Na etapa de segmentação de imagens, quando os índices foram aplicados, o índice ExG+SML obteve o melhor resultado, ao contar $96,4 \%$ das mudas com índice de acerto de $83 \%$.

Como contribuições práticas para o agricultor, este estudo possibilitou a conscientização da importância de prever a estimativa da colheita para melhor planejar a negociação da produção, os plantios posteriores e a busca por recursos para aumentar a área mecanizada da cultura. Também foi possível perceber a necessidade de preparar as linhas de plantio, de melhorar o preparo do solo e a irrigação.

\section{References}

Anguiano-Morales, M., Corral-Martínez, L. F., Trujillo-Schiaffino, G., Salas-Peimbert, D. P., García-Guevara, A. E. (2018) "Topographic investigation from a low altitude unmanned aerial vehicle", Optics and Lasers in Engineering: 110, 63-71.

CONTAG - Confederação Nacional dos Trabalhadores na Agricultura (2017) "Development of family farming", Anais do Congresso Nacional de Trabalhadores Rurais Agricultores e Agricultoras Familiares, Março, pp. 36-39, Cidade Gráfica.

EMBRAPA (2018) “Análises gráficas dos principais produtos agropecuários do Estado do Pará", https://www.embrapa.br/congresso-de-mandioca-2018/mandioca-emnumeros. 
FAPESPA - Fundação Amazônia de Amparo a Estudos e Pesquisas do Pará (2015) "Boletim Agropecuário do Estado do Pará", http://www.fapespa.pa.gov.br/sites/default/files/Boletim_Agropecuario_do_Estado_d o_Para_2015.pdf.

Feng, A., Zhang, M., Sudduth, K. A., Vories, E. D., Zhou, J. (2019) “Cotton yield estimation from UAV-based plant height", Trans. of the ASABE: 62(2), 393-404.

Gnädinger, F.E, Schmidhalter, U. (2017) "Digital Counts of Maize Plants by Unmanned Aerial Vehicles (UAVs)", Remote Sensing: 9(6), 544-559.

Hamuda, E., Glavin, M., Jones, E. (2016). "A survey of image processing techniques for plant extraction and segmentation in the field", Computers and Electronics in Agriculture: 125, pages 184-199.

Hague, T. Tillet, N. D., Wheeler, H. (2006), "Automated crop and weed monitoring in widely spaced cereals”, Precision Agriculture: 7, 21-32. Springer.

Hunt, E. R., Cavigelli, M., Daughtry, C. T., McMurtrey, J. Walthall, S. L. (2005), "Evaluation of digital photography from model aircraft for remote sensing of crop biomass", Precision Agiculture: 6(4), 359-378, Springer.

Kataoka, T., Kaneko, T., Okamoto, H., Hata, S.(2003), "Crop growth estimation system using machine vision", In Proceedings 2003 IEEE/ASME International Conference on Advanced Intelligent Mechatronics (AIM 2003): Vol. 2, pp. b1079-b1083. IEEE.

Ghosal, S., Zheng, B., Chapman, S. C., Potgieter, A. B., Jordan, D. R., Wang, Singh, A. K., Singh, A., Ganapathysubramanian, B., Hirafuji, A., Ninomiya, S. (2019) "A weakly supervised deep learning framework for sorghum head detection and counting”, Plant Phenomics: pages 1-14.

Guerrero, J. M., Pajares, G., Montalvo, M., Romeo, J., Guijarro, M. (2012) "Support vector machines for crop/weeds identification in maize fields", Expert Systems with Applications: 39(12), pages 11149-11155.

Guijarro, M., Pajares, G., Riomoros, I., Herrera, P. J., Burgos-Artizzu, X. P., Ribeiro, A. (2011) "Automatic segmentation of relevant textures in agricultural images", Computers and Electronics in Agriculture: 75(1), pages 75-83.

McCraine, C. D., Samiappan, S., Czarnecki, J. M. P., Dodds, D. M. (2019) "Plant density estimation and weeds mapping on row crops at emergence using low altitude UAS imagery", In Autonomous Air and Ground Sensing Systems for Agricultural Optimization and Phenotyping: IV(11008), p. 110080Y. International Society for Optics and Photonics.

Meyer, G. E., Camargo-Neto, J., Jones, D. D., Hindman, T. W. (2004) "Intensified fuzzy clusters for classifying plant, soil, and residue regions of interest from color images", Computers and electronics in agriculture: 42(3), pages 161-180.

Meyer, G. E., Camargo Neto, J. (2008) "Verification of color vegetation indices for automated crop imaging applications", Computers and electronics in agriculture: vol. 63, pages 282-293. Elsevier.

Moorthy, S., Boigelot, B., Mercatoris, B. C. N. (2015) "Effective segmentation of green vegetation for resource-constrained real-time applications", In Precision agriculture: 15, pages 93-98. Wageningen Academic Publishers. 
Netto, A. F. A., Martins, R. N., de Souza, G. S. A., Araújo, G. D. M., de Almeida, S. L. H., Capelini, V. A. (2018), "Segmentation of RGB images using different vegetation indices and thresholding methods", Nativa: Pesquisas Agrárias e Ambientais, 6(4), 389-394.

Otsu, N. (1979) "A threshold selection method from gray-level histogram". IEEE Transactions on Systems Man and Cybernetics: 9(1), pages 62-66.

Riddler, T. W., Calvard, S. (1978), "Picture thresholding using an iterative selection method", IEEE trans syst Man Cybern: 8(8), 630-632.

SEAD - Secretaria Especial de Agricultura Familiar e do Desenvolvimento Agrário (2017) "Brasil: 70\% dos alimentos que vão à mesa dos brasileiros são da agricultura familiar", http://www.mda.gov.br/sitemda/noticias/brasil-70-dos-alimentos-quev\%C3\%A3o-\%C3\%A0-mesa-dos-brasileiros-s\%C3\%A3o-da-agricultura-familiar, acessado em 11/08/2019.

Schalkoff, R.J. (1989) Digital Image Processing and Computer Vision, Wiley, $1^{\text {th }}$ edition.

Soares, G. A. (2017) "Identificação de linhas de plantio por meio da transformada de Hough", Trabalho de conclusão de curso, Faculdade de Computação da Universidade Federal de Uberlândia, Minas Gerais.

Varela, S., Dhodda, P. R., Hsu, W. H., Prasad, P. V., Assefa, Y., Peralta, N. R., T., Griffin, A., Sharda, A., Ferguson, Ciampitti, I. A. (2018) "Early-season stand count determination in corn via integration of imagery from unmanned aerial systems (UAS) and supervised learning techniques", Remote Sensing: 10(2), 343.

Woebbecke, D. M., Meyer, G. E., Von Bargen, K., Mortensen, D. A. (1993) “ Plant species identification, size, and enumeration using machine vision techniques on near-binary images", In Optics in Agriculture and Forestry: (1836), 208-219.

Woebbecke, D. M., Meyer, G. E., Von Bargen, K., Mortensen, D. A. (1995) "Color indices for weed identification under various soil, residue, and lighting conditions", Transactions of the ASAE: (38), 259-269.

Zack, G. W., Rogers, W. E., Latt, S. A. (1977). "Automatic measurement of sister chromatid exchange frequency", Journal of Histochemistry \& Cytochemistry: 25(7), 741-753. 